\title{
RISCOS AMBIENTAIS EM UM SERVIÇO DE ATENÇÃO SECUNDÁRIA À SAÚDE
}

\author{
Juliana Trebi Penatti ${ }^{1}$, Tatiane Bonametti Veiga² ${ }^{2}$ Adriana Aparecida Mendes³, Ana Paula Milla dos Santos ${ }^{4}$, \\ Angela Maria Magosso Takayanagui ${ }^{5}$
}

RESUMO: Esta pesquisa foi realizada com o objetivo de identificar e quantificar os riscos ambientais em serviço de atenção secundária à saúde, levando-se em consideração o nível de escolaridade e as atividades desenvolvidas pelos participantes do estudo que compõem a equipe multidisciplinar, visando contribuir para o alcance de melhores condições de saúde ocupacional. Trata-se de uma pesquisa descritiva e exploratória, de abordagem quali-quantitativa. A partir do perfil e atuação profissional dos participantes foi possível identificar o conhecimento e a percepção desses sobre os riscos, bem como identificar e quantificá-los. Os resultados evidenciam preocupação sobre questões relativas às condições de trabalho e aos riscos ambientais presentes em suas atividades diárias e permitem suscitar novas abordagens para o trabalho gerencial, principalmente de caráter educacional e estrutural no serviço.

DESCRITORES: Saúde ambiental; Saúde ocupacional; Riscos ambientais.

\section{ENVIRONMENTAL RISKS IN A SERVICE FOR SECONDARY CARE FOR HEALTH}

ABSTRACT: This research was undertaken with the objective of identifying and quantifying the environmental risks in a service for secondary care for health, taking into account the level of schooling of, and the activities carried out by, the participants of the study, who made up the multidisciplinary team, with a view to contributing to the achieving of better occupational health conditions. This is descriptive and exploratory research, with a quali-quantitative approach. Based on the participants' professional profiles and functions, it was possible to identify the knowledge and perceptions that these have on the risks, as well as identifying and quantifying them. The results evidence concern about questions relevant to working conditions and to the environmental risks present in their daily activities, and allow one to generate new approaches to the managerial work, principally of an educational and structural character in the work.

DESCRIPTORS: Environmental health; Occupational health; Environmental risks.

\section{RIESGOS AMBIENTALES EN UN SERVICIO DE ATENCIÓN SECUNDARIA A LA SALUD}

RESUMEN: Esta investigación fue realizada con el objetivo de identificar y cuantificar los riesgos ambientales en servicio de atención secundaria a la salud, tomándose en cuenta el nivel de escolaridad y las actividades desarrolladas por los participantes del estudio que componen el equipo multidisciplinar, con fines de contribuir para el alcance de mejores condiciones de salud ocupacional. Es una investigación descriptiva y exploratoria, de abordaje cuali-cuantitativo. Con base en el perfil y en la actuación profesional de los participantes, fue posible identificar el conocimiento y la percepción de estes acerca de los riesgos, así como identificar y cuantificarlos. Los resultados evidencian preocupación sobre cuestiones acerca de las condiciones de trabajo y de los riesgos ambientales presentes en sus actividades diarias y permiten suscitar nuevos abordajes para el trabajo administrativo, principalmente de carácter educacional y estrutural en el servicio.

DESCRIPTORES: Salud ambiental; Salud ocupacional; Riesgos ambientales.

${ }^{1}$ Enfermeira. Mestranda em Ciências pelo Programa Enfermagem em Saúde Pública da Escola de Enfermagem de Ribeirão Preto da Universidade de São Paulo-EERP USP. Membro do Grupo de Pesquisa Interinstitucional de Estudos da Problemática dos Resíduos de Serviços de Saúde - GIERSS. ${ }^{2}$ Engenheira Civil. Mestre em Ciências. Doutoranda em Ciências pelo Programa Enfermagem em Saúde Pública da EERP USP. Membro do GIERSS. ${ }^{3}$ Enfermeira. Mestre em Desenvolvimento Regional e Meio Ambiente. Doutoranda em Ciências pelo Programa Enfermagem em Saúde Pública da EERP USP. Membro do GIERSS.

${ }^{4}$ Bióloga. Mestre em Ecologia. Doutoranda em Ciências pelo Programa Enfermagem em Saúde Pública da EERP USP. Membro do GIERSS. ${ }^{5}$ Enfermeira. Doutora em Enfermagem. Professora do Departamento de Enfermagem Materno-Infantil e Saúde Pública da EERP USP. Responsável pelo Laboratório de Saúde Ambiental da EERP USP. Coordenadora do Grupo de Pesquisa GIERSS.

Autor correspondente:

Recebido: 20/07/2012

Juliana Trebi Penatti

Aprovado: 11/03/2013

Universidade de São Paulo.

Av. Bandeirantes, 3900 - 14040-902 - Ribeirão Preto-SP-Brasil

E-mail: julianatrebi@yahoo.com.br 


\section{INTRODUÇÃO}

A Saúde Ambiental tornou-se um dos principais pilares do desenvolvimento sustentável; no entanto, o desenvolvimento econômico, social e tecnológico é limitado, além da possibilidade de expor a população a diversos riscos ambientais (RA), que podem influenciar negativamente o processo saúde-doença ${ }^{(1)}$.

Diante do estilo de vida atual, embasado em um crescente processo de desenvolvimento socioeconômico e também no paradigma de consumo/poder, diversos setores, tanto do processo produtivo, quanto do processo de trabalho em geral, podem estar expostos a diferentes riscos e ameaças à saúde. Em todas as atividades humanas, inclusive no ambiente de trabalho, há RA que podem estar relacionados à saúde, à segurança ou afetar as condições ambientais dos espaços onde ocorrem essas atividades.

São grandes os desafios e inquietações pelos quais passam a sociedade moderna, frente aos diagnósticos ambientais e seus efeitos desencadeantes na saúde da população, em razão do crescente aumento da complexidade dos problemas ambientais e de saúde ${ }^{(2)}$.

Assim, suprir as necessidades da geração atual, sem comprometer a capacidade do meio ambiente para atendimento das necessidades das futuras gerações, de acordo com os princípios da sustentabilidade, é uma das justificativas para se alcançar melhores condições da saúde ambiental ${ }^{(3)}$. RA são aqueles causados por agentes físicos, químicos ou biológicos, e quando presentes nos ambientes de trabalho são capazes de causar danos à saúde do trabalhador em função de sua natureza e da concentração, intensidade ou tempo de exposição ${ }^{(4)}$. De acordo com sua natureza, os RA são classificados em cinco grupos: físicos, químicos, biológicos, ergonômicos e de acidentes, segundo a legislação brasileira $^{(5-6)}$. Na área da saúde, os resíduos gerados em unidades de atenção à saúde podem apresentar interferência direta na saúde dos trabalhadores. Assim, pode-se também incluir os resíduos de serviços de saúde (RSS) gerados em diferentes serviços de saúde, uma vez que podem conter agentes físicos, químicos e radioativos ${ }^{(7)}$.

No Brasil, a Norma Regulamentadora 9 estabelece a obrigatoriedade de identificar e avaliar RA existentes, ou que venham a existir no ambiente de trabalho, e que possam prejudicar a integridade da saúde dos trabalhadores $^{(5-6)}$. Modelos de identificação e avaliação de riscos englobam uma série de procedimentos que devem ter como objetivo levantar o potencial de danos à saúde ocasionados pela exposição de trabalhadores a agentes ambientais; essas avaliações servem de embasamento para o controle e prevenção desse tipo de exposição ${ }^{(8)}$. Em uma abordagem de avaliação de riscos considera-se necessária a priorização da identificação desses riscos pelos trabalhadores, possibilitando o envolvimento ativo desses no processo para melhor compreensão de sua realidade ${ }^{(9)}$.

Em Estabelecimentos de Assistência à Saúde (EAS), considerando a natureza dos serviços prestados e os aspectos das atividades peculiares, torna-se elevada a probabilidade de riscos e acidentes envolvendo o pessoal que neles trabalham ${ }^{(10)}$, sendo necessário uma avaliação dos RA envolvidos. É importante destacar que os acidentes ocupacionais podem estar relacionados tanto às atividades laborais, quanto às condições de trabalho e aos trabalhadores ${ }^{(11)}$.

Além da avaliação e controle dos RA, há estudos que abordam a educação dos trabalhadores da área da saúde como uma importante ferramenta para a prevenção de acidentes envolvendo RA, tal como os riscos biológicos ${ }^{(12)}$. No Brasil, em estabelecimentos de atenção secundária à saúde, como em Unidades Mistas de Saúde (UMS), destaca-se a grande diversidade de agentes físicos, químicos e biológicos, presentes e que geralmente não diferem muito de instituições hospitalares.

As UMS são destinadas à prestação de atendimento em atenção básica e integrada de saúde, de forma programada ou não, nas especialidades básicas, podendo oferecer assistência por profissionais especialistas, e contar com unidade de internação. Nesses serviços deve haver disponível serviço de urgência e emergência, bem como assistência permanente. Essas Unidades também possuem características de hospital local de pequeno porte, estando sob administração única ${ }^{(13)}$. Adicionalmente, há grande diversidade de agentes físicos, químicos, biológicos e ergonômicos também presentes em UMS, o que exige um adequado controle de RA nesses serviços. Destaca-se que estudos sobre esses riscos ligados à saúde dos trabalhadores, frequentemente são realizados em serviços de grande porte, do tipo hospitalar, e na maioria das vezes, com foco voltado para a equipe de Enfermagem.

Nesse sentido, essa pesquisa justificou-se pela necessidade de se conhecer a realidade de exposição a RA, envolvendo a equipe multidisciplinar de uma UMS, com vistas à proposição de melhores condições de saúde ocupacional, e teve por objetivos identificar e quantificar os RA a partir da percepção dos trabalhadores. 


\section{MÉTODO}

Trata-se de uma pesquisa descritiva e exploratória de abordagem quali-quantitativa, tomando-se como referencial teórico a legislação nacional sobre saúde e segurança ocupacional ${ }^{(5-6)}$. A pesquisa foi realizada na Unidade Mista de Saúde de um município do interior do Estado de São Paulo, a qual representa o único serviço de saúde disponível para uma população estimada em 8.346 habitantes.

Utilizou-se um instrumento composto por perguntas semiestruturadas e um check-list, elaborado pelos autores, para uma única entrevista previamente agendada com os participantes, realizada no local de estudo e durante o horário de trabalho. As entrevistas foram realizadas durante os meses de janeiro e fevereiro de 2012. Esse instrumento abrangeu questões referentes à identificação dos participantes e atividade profissional, bem como sua percepção sobre RA, com a identificação e quantificação de possíveis riscos referidos.

Os critérios de inclusão adotados para a constituição do número de participantes do presente estudo foram: ser trabalhador e fazer parte da equipe permanente da UMS selecionada. Foi levado em consideração o nível de escolaridade e as diferentes atividades profissionais desenvolvidas. O critério de exclusão dos participantes foi ausência no serviço no período da coleta de dados por falta, férias ou por diferentes tipos de licenças. Não houve necessidade de reagendamento das entrevistas marcadas.

Assim, foram selecionados por sorteio cinco participantes, pertencentes a diferentes categorias profissionais, como médico, enfermeiro, recepcionista, motorista e técnico em enfermagem, que concordaram em participar da pesquisa, assinando um Termo de Consentimento Livre e Esclarecido, aprovado pelo Comitê de Ética em Pesquisa da Escola de Enfermagem de Ribeirão Preto da Universidade de São Paulo, protocolo n. 1226/2010.

\section{RESULTADOS}

Com a realização deste estudo obteve-se dados que foram divididos em três partes: perfil dos sujeitos e atuação profissional; conhecimento e percepção sobre RA; identificação e quantificação dos RA.

\section{Perfil dos participantes e atuação profissional}

Em relação às categorias profissionais, foi selecionado um participante de cada categoria escolhida para este estudo, visando-se obter diferentes visões dentro da equipe de trabalho. Quanto à faixa etária dos participante, a maioria (80\%) tinha entre 20 e 30 anos e $20 \%$ entre 40 e 50 anos.

A maior parte dos participantes (60\%) pertencia ao gênero feminino. No que se refere à formação dos entrevistados, $60 \%$ possuíam ensino superior completo, seguida por $40 \%$ com ensino médio completo. Quanto ao tempo de trabalho na função, quatro participantes (80\% do total) referiram desempenhar atividades relativas às suas funções atuais por um período entre um a três anos e um por mais de 10 anos.

No que diz respeito à carga horária de trabalho realizada, levando-se em conta a soma da carga horária de todos os empregos, $60 \%$ dos entrevistados relataram ter carga horária semanal de trabalho entre 40 e 70 horas, 20\% relataram carga horária semanal de 36 horas e igual percentual não informou a carga horária desempenhada semanalmente.

\section{Conhecimento e percepção sobre riscos ambientais}

A segunda parte da entrevista abrangeu dados sobre o conhecimento e a percepção dos participantes sobre RA presentes no serviço. Quando perguntado aos entrevistados sobre riscos em qualquer tipo de ambiente de trabalho, todos relataram já ter ouvido falar sobre o tema e sua importância; e quando questionados sobre riscos dentro do ambiente de trabalho selecionado para o estudo, todos afirmaram haver riscos naquele ambiente.

\section{Identificação e quantificação dos riscos ambientais}

Em relação a esse item, pelo check-list utilizado, pode-se verificar a existência de diferentes tipos de riscos no ambiente de trabalho.

Para os riscos físicos, destaca-se que $80 \%$ dos participantes consideraram ruído como um risco presente no ambiente de trabalho; $100 \%$ consideraram as radiações não ionizantes; $80 \%$ citaram calor como um risco ambiental de alta intensidade; a respeito de frio, apenas $25 \%$ consideraram este fator como um risco de alta intensidade; a umidade foi considerada como risco por $60 \%$ dos participantes.

Em relação aos riscos químicos, $100 \%$ dos entrevistados consideraram as poeiras como um risco ambiental presente na Unidade de Saúde, apontando como causa a localização do serviço, situado em área cercada por propriedades com extensas áreas de terra e sem pavimentação em vários trechos. 
Quando perguntados sobre a presença de substâncias tóxicas e produtos químicos, apenas $20 \%$ dos entrevistados consideraram esses fatores como um risco presente no local, referindo preocupação com o manuseio de produtos quimioterápicos, detergentes enzimáticos, antibióticos e eritropoetina. Houve um relato de descarte desses resíduos, contendo substâncias químicas, em recipientes destinados ao descarte de resíduos biológicos ou em caixas rígidas destinadas ao descarte de resíduos perfurocortantes, sem nenhum tratamento prévio. Isso revela acondicionamento inadequado de RSS que acaba resultando em inadequação de seu tratamento.

Dentre os riscos biológicos, vírus, bactérias e fungos foram considerados como RA presente por $100 \%$ dos participantes. Protozoários e parasitas representam risco apenas para $40 \%$ dos entrevistados. Destaca-se que um deles deu como exemplo de parasita, mosquitos presentes no ambiente, demonstrando um conceito errôneo sobre a definição de parasitas.

Em relação a riscos de acidentes, para $60 \%$ dos participantes a condição de Equipamentos de Proteção individual (EPI) inadequados representa RA, sendo que $66,7 \%$ consideraram esse fator como um risco de média intensidade e 33,3\% um risco de baixa intensidade. Além de relatos de EPI inadequados, como luvas que rasgam com facilidade, a falta de EPI também foi citada, como exemplo falta de máscaras com filtro, aventais de plástico e óculos de proteção individual.

\section{DISCUSSÃO}

Os participantes deste estudo foram, em sua maioria, trabalhadores do gênero feminino; e quando analisadas somente as categorias de técnico de enfermagem e enfermeiro, $100 \%$ desses pertenciam a esse gênero. O corpo de trabalhadores da enfermagem, no Brasil, é representado, no geral, por trabalhadores do gênero feminino em todas as suas categorias profissionais, segundo o modelo profissional da enfermagem instituída no país ${ }^{(14)}$.

No que diz respeito à carga horária desempenhada pelos trabalhadores, a maioria revelou desempenhar uma carga horária elevada. Um estudo realizado em um hospital de Recife, em 2011, com 44 enfermeiros, demonstrou que a carga horária desempenhada por trabalhadores da área da saúde pode estar relacionada a um maior desgaste físico e mental, levando-se em consideração que o tempo destinado ao trabalho é inversamente proporcional ao tempo destinado ao $\operatorname{lazer}^{(14)}$.
Quanto à identificação e quantificação dos RA, a maioria destacou a presença de ruídos, radiações não ionizantes e temperatura como principais RA, corroborando as considerações da Organização Internacional do Trabalho que aponta como principais fatores de risco físico para os trabalhadores da área da saúde, acrescidos da iluminação ${ }^{(15)}$.

Em relação à presença de substâncias tóxicas e produtos químicos no ambiente de trabalho, houve apenas um relato desses fatores ligados a RA nos seus espaços de trabalho. Nessa mesma direção, há um estudo revelando que os riscos químicos presentes em serviços de saúde são pouco valorizados, em termos da importância de seus danos, sendo considerado de difícil avaliação devido ao grande número de substâncias empregadas em diferentes procedimentos ${ }^{(16)}$.

Todos os entrevistados consideraram os riscos biológicos como um tipo de risco ambiental presente no local onde foi desenvolvido o estudo. Isto vai ao encontro de resultados da literatura científica sobre estudos realizados com trabalhadores da saúde, os quais ressaltam que os riscos biológicos representam os principais geradores de periculosidade e insalubridade aos trabalhadores de serviços de atenção à saúde, além de serem responsáveis pela transmissão de patógenos, necessitando de medidas para a prevenção e minimização desses riscos ${ }^{(10,17)}$.

No que diz respeito aos EPI, sabe-se que o uso adequado desses equipamentos, durante as atividades desenvolvidas por equipes da área da saúde, depende do fornecimento e manutenção de equipamentos pela instituição, bem como da predisposição do trabalhador para sua utilização, sendo indispensáveis na prevenção de acidentes. Porém, é sabido da problemática relacionada à resistência de trabalhadores para utilização de EPI oferecidos nos serviços ${ }^{(18)}$.

\section{CONCLUSÕES}

Os resultados permitiram conhecer, embora em perspectiva restrita, a realidade dos RA presentes no cenário de trabalho, por meio do relato de um grupo de trabalhadores. Um fator que pode ser considerado positivo foi a preocupação manifesta pelos participantes do estudo sobre questões relativas às condições de trabalho e percepção dos riscos presentes em suas atividades diárias. Diante do exposto, pode-se concluir que os RA em serviços de atenção secundária à saúde podem representar perigo à saúde dos trabalhadores; porém esta situação é pouco abordada na literatura, 
estando bem documentados estudos envolvendo RA em serviços de atenção terciária à saúde.

\section{REFERÊNCIAS}

1. Periago MR, Galvão LA, Corvalan C, Finkelman J. Saúde ambiental na América Latina e no Caribe: numa encruzilhada. Saude soc. 2007;16(3):14-9.

2. Freitas CM, Porto MF. Saúde, Ambiente e sustentabilidade. Rio de Janeiro: Fiocruz; 2006. p.120.

3. Conferência da Organização das Nações Unidas sobre Meio Ambiente e Desenvolvimento. Agenda 21. Curitiba: IPARDES; 2001. p. 260.

4. Hökerberg Y, Santos MA, Passos SR, Rozemberg B, Cotias PM, Alves L et al. O processo de construção de mapas de risco em um hospital público. Ciênc. saúde colet. 2006;11(2):503-13.

5. Ministério da Saúde (BR). Portaria n. 3.214, de 08 de junho de 1978. Aprova as Normas Regulamentadoras NR - do Capítulo V, Título II, da Consolidação das Leis do Trabalho, relativas à Segurança e Medicina do Trabalho. Diário Oficial da União, Brasília, DF, 06 jul. 1978.

6. Ministério da Saúde(BR). Portarian.25, de29 de dezembro de 1994. Aprova o texto da Norma Regulamentadora n. 9 do Ministério do Trabalho e Emprego. Diário Oficial da União, Brasília, DF, 30 dez. 1994.

7. Takayanagui AMM. Gerenciamento de resíduos sólidos de serviços de saúde. In: Philippi Jr A. Saneamento, saúde e ambiente: fundamento para um desenvolvimento sustentável. São Paulo: Manole; 2005. p. 323-74.

8. Porto MFS, Freitas CM. Análise de riscos tecnológicos ambientais: perspectivas para o campo da saúde do trabalhador. Cad. Saúde Pública. 1997;13(2):109-18.

9. Santos LA, Guimarães KR, Teodoro VA, Bevilacqua PD. Biossegurança em ambiente hospitalar/laboratorial e o uso das técnicas de DRP: experiência no Departamento de Veterinária da Universidade Federal de Viçosa (DVT/UFV). Seminário Nacional de Saúde e Ambiente no Processo de Desenvolvimento. Saúde e Ambiente no Processo de Desenvolvimento. (Série Fiocruz Eventos Científicos 2). Rio de Janeiro: Fiocruz; 2000. p. 181.

10. Canini SRMS, Gir E, Hayashida M, Machado ASM. Acidentes perfurocortantes entre trabalhadores de enfermagem de um hospital universitário do interior paulista. Rev. Latino-Am. Enfermagem. 2002;10(2):172-8.
11. Izidoro JS, Iwamoto HH, Camargo FC. Delineamento dos acidentes de trabalho em instituições de saúde de uma microrregião de Minas Gerais. Cogitare enferm. 2010;15(3):521-7.

12. Soares LG, Labrocini LM, Maftum MA, Sarquis LMM, Kirchhof AL. Risco biológico em trabalhadores de enfermagem: promovendo a reflexão e a prevenção. Cogitare enferm. 2011;16(2):261-7.

13. Ministério da Saúde (BR). Manual Técnico do Cadastro Nacional de Estabelecimentos de Saúde. Brasília; 2006.

14. Santos TMB, Frazão IF, Ferreira DMA. Estresse ocupacional em enfermeiros de um hospital universitário. Cogitare enferm. 2011;16(1):76-81.

15. Organização Internacional do Trabalho. Convenções da OIT. [Internet] [acesso em 25 mai 2012]. Disponível: http://www.oitbrasil.org.br/node/500

16. Prista J, Uva AS. Exposição profissional a agentes químicos: os indicadores biológicos na vigilância de saúde dos trabalhadores. Saúde \& Trabalho. 2003;(4):5-12.

17. Garner JS. Guideline for isolation precautions in hospitals. Infect. control hosp. epidemiol. 1996;1(17):54-80.

18. Penatti JT, Takayanagui AMM. Exposição ocupacional ao glutaraldeído em um hospital-escola do Interior de São Paulo, 2007 - 2008. [trabalho de conclusão de curso]. Ribeirão Preto (SP): Universidade de São Paulo; 2008. 